CLINICAL STUDY

\title{
Investigation of adrenal functions in patients with idiopathic hyperandrogenemia
}

\author{
Hulusi Atmaca, Fatih Tanriverdi ${ }^{1}$, Kursad Unluhizarci ${ }^{1}$, Fahri Bayram ${ }^{1}$ and Fahrettin Kelestimur ${ }^{1}$ \\ Department of Endocrinology, Zonguldak Karaelmas University Medical School, Zonguldak, Turkey and ${ }^{1}$ Department of Endocrinology, Erciyes University \\ Medical School, Kayseri, Turkey \\ (Correspondence should be addressed to F Kelestimur; Email: fktimur@erciyes.edu.tr)
}

\begin{abstract}
Objective: Some patients with hyperandrogenemia had no identifiable cause, which was named as idiopathic hyperandrogenemia (IHA). The role of the adrenal glands in these patients was investigated. Design: Clinical study in patients with IHA at the Endocrinology Department of a University Hospital. Patient(s): In this study, 26 pre-menopausal women with IHA and 20 healthy women were included. Basal hormonal investigations, ACTH test and a $75 \mathrm{~g}$ oral glucose tolerance test (OGTT) were performed. Basal levels of total testosterone, free testosterone, androstenedione $\left(\mathrm{A}_{4}\right)$, sex hormone-binding globulin, DHEA sulfate (DHEAS), cortisol, 17-hydroxyprogesterone (17-OHP), 11-deoxycortisol (11-S) and ACTH-stimulated levels of cortisol, $\mathrm{A}_{4}$, DHEAS, 17-OHP, and 11-S were measured. Additionally, glucose and insulin responses to OGTT were obtained.

Results: The patients and the control subjects had similar age and body mass index. Peak and area under the curve (AUC) responses of 11-S $(P<0.05)$, DHEAS $(P<0.005)$, and $A_{4}$ (peak, $P<0.005$; AUC, $P<0.001)$ to ACTH test were significantly higher in the patients with IHA than in the control subjects. There was a significant correlation between the basal DHEAS levels, peak 11-S, and $\mathrm{AUC}_{11-\mathrm{s}}$, in response to ACTH-stimulation test in patients with IHA $(P<0.005, r, 0.6)$. Four $(16.6 \%)$ patients with IHA had glucose intolerance.

Conclusion: Our data suggest that adrenal androgen excess may play an important role in patients with IHA and these patients exhibit increased prevalence of glucose intolerance.
\end{abstract}

European Journal of Endocrinology 155 307-311

\section{Introduction}

Hirsutism is a common clinical condition in women during reproductive age and characterized by excessive growth of terminal hair in the androgen-sensitive skin regions. It affects $5-8 \%$ of the whole female population of fertile age and may be associated with underlying endocrine and metabolic disturbances or the initial manifestation of an androgen secreting tumor (1). Some drugs, polycystic ovary syndrome (PCOS), enzyme defects in adrenal steroid biosynthesis, Cushing's syndrome, acromegaly, ovarian tumors, and adrenal tumors may be the underlying causes or it may be idiopathic (1-3). PCOS and idiopathic hirsutism account for most of the cases of hirsutism. Azziz et al. (1) reported that $6.7 \%$ of the patients with androgen excess in 873 consecutive patients had hirsutism with normal ovulation. We recently investigated 168 women with hirsutism to determine the various causes of hirsutism and their frequencies in a Turkish population and found that $17.4 \%$ of the patients had hyperandrogenemia with no identifiable cause, which was named idiopathic hyperandrogenemia (IHA) (3).
The objective of the present study was to investigate the adrenal gland functions in patients with IHA in order to understand whether there is an adrenal contribution to the high androgen levels in the circulation. We have also evaluated the glucose tolerance status in these patients in order to see whether IHA is also associated with insulin resistance like some other androgen excess disorders.

\section{Patients and methods}

\section{Subjects}

In this study, 26 women with IHA and 20 healthy Turkish women were included. The study was approved by the local Ethics Committee and informed consent was obtained from all subjects. All patients had hirsutism (modified Ferriman-Gallwey score, > 8) (4), regular ovulatory menstrual cycles (intermenstrual interval 21-35 days), and hyperandrogenemia. Hyperandrogenemia was defined as an androgen value above the normal reference range (i.e. total testosterone $>80 \mathrm{ng} / \mathrm{dl}$ and/or free testosterone $>3.9 \mathrm{pg} / \mathrm{ml}$ and/or 
androstenedione $\left(\mathrm{A}_{4}\right)>2.99 \mathrm{ng} / \mathrm{ml}$ and/or DHEA sulfate $($ DHEAS $)>5070(\mathrm{ng} / \mathrm{ml}))$. Thyroid dysfunction, hyperprolactinemia, PCOS, Cushing's disease, and adrenal/ ovarian tumors were excluded by appropriate tests and ultrasonography of the ovaries and the adrenal glands. Patients with regular menses and normal ovulation but polycystic ovaries on ultrasound examination were also excluded. Ovulation was confirmed by the measurement of day 22-24 serum progesterone level ( $>8 \mathrm{nmol} / \mathrm{l})$. The adrenocorticotropin (ACTH) stimulation test (0.25 mg Synacthen, Nürnberg, Germany) was performed in all subjects to assess adrenocortical responsiveness. An ACTH-stimulated 17-hydroxyprogesterone (17-OHP) level $>10 \mathrm{ng} / \mathrm{ml}$ was considered the criterion of non-classic adrenal hyperplasia (NCAH) due to 21-hydroxylase deficiency (5). The diagnosis of $11 \beta$-hydroxylase deficiency was made if the 11-deoxycortisol (11-S) response to ACTH stimulation exceeded the 95 th percentile of controls $(6-8)$ by threefold. The presence of insulin resistance was investigated by using basal insulin levels, oral glucose tolerance test (OGTT), and homeostasis model assessment (HOMA) score in both groups. The estimate of insulin resistance by HOMA score was calculated with the formula: fasting serum insulin $(\mu \mathrm{U} / \mathrm{ml}) \times$ fasting plasma glucose $(\mathrm{mmol} / \mathrm{l}) / 22.5$ (9). Glucose tolerance was evaluated by using the criteria of the American Diabetes Association, and impaired glucose tolerance (IGT) was defined as a 2-h post-load glucose of $140 \mathrm{mg} / \mathrm{dl}$ or greater and less than $200 \mathrm{mg} / \mathrm{dl}$ (10). The glucose and insulin responses to the OGTT were expressed as the area under the curve (AUC) estimated by the trapezoidal rule. Cortisol, 17-OHP, 11-S, A 4 , and DHEAS responses to ACTH were also expressed as AUCs. None of the subjects in the patient or control groups had received any hormonal medication at least for 6 months before the study.

\section{Study protocol}

All the patients and the control women completed a standardized history form and underwent a full physical examination. Weight (in kilograms) and height (in meters) were measured to calculate body mass index (BMI). Serum A4, DHEAS, cortisol, 17-OHP, and 11-S levels were measured before, 30 and 60 min after ACTH stimulation. All the hormonal analyses were performed in the follicular phase of their cycles. The OGTT was performed after 10-12 h of fasting between 0800 and 1000 h. A $300 \mathrm{~g}$ carbohydrate diet was given for 3 days before the OGTT. After a basal blood sample was obtained, a $75 \mathrm{~g}$ glucose load was administered orally and blood samples were obtained at 30-min intervals for $2 \mathrm{~h}$ for the measurement of glucose and insulin.

\section{Hormonal assays}

The serum samples were stored at $-20{ }^{\circ} \mathrm{C}$ until assayed. Serum $\mathrm{A}_{4}$ (DSL-3800, Webster, TX, USA), testosterone (Biosource, Nivelles, Belgium), cortisol (DSL-2100), free testosterone (Biosource, Nivelles, Belgium), 11-S (ICN Pharmaceuticals, Costa Mesa, CA, USA), 17-OHP (DSL-5000), and DHEAS (DSL-3500) levels were measured by RIA; serum sex hormone-binding globulin (SHBG) (Zentech, Angleur, Belgium) and insulin (Biosource, Nivelles, Belgium) levels were measured by IRMA; and serum folliclestimulating hormone (FSH), luteinizing hormone (LH), and estradiol levels (ACS:180, Bayer) were determined by an automated chemiluminescence system, using commercial kits. The intra- and interassay coefficients of variation were: 2.8 and $7 \%$ for $\mathrm{A}_{4}, 4.4$ and $4.8 \%$ for testosterone, 11.1 and $11.5 \%$ for cortisol, 4.3 and $7.8 \%$ for free testosterone, 4.3 and $11.6 \%$ for $11-S, 9.5$ and $10.8 \%$ for 17-OHP, 6.3 and $9.9 \%$ for DHEAS, 5.2 and $5.8 \%$ for SHBG, 1.6 and $6.1 \%$ for insulin, 2.8 and $4.6 \%$ for $\mathrm{FSH}, 5$ and $6.2 \%$ for LH, 9.9 and $11.8 \%$ for E2 respectively.

\section{Statistical analysis}

Results are expressed as means \pm s.D. in the text and tables. The results of the women with IHA and healthy women were compared by Mann-Whitney U-test and Spearmen $(r)$ coefficient for correlation. $P<0.05$ was considered statistically significant.

\section{Results}

Patients and the control subjects did not differ in mean age and BMI. The modified Ferriman-Gallwey score was significantly higher in the patients with IHA than in the control subjects $(P<0.001)$. None of the patients had any history of premature adrenarche/pubarche. Nine of them were married and did not have any infertility problems. Clinical characteristics of the patients with IHA and control subjects are shown in Table 1. Basal FSH and LH levels were similar between the patients and control subjects. The patients with IHA had at least one significantly higher serum androgen level than the control group $(P<0.05)$. Basal 17-OHP, 11-S, and SHBG levels were not significantly different in the patients with IHA compared with the controls (Table 1). ACTH-stimulated peak 17-OHP and cortisol levels were similar between the two groups. Peak and AUC responses of $11-\mathrm{S}(P<0.05)$, DHEAS $(P<0.005)$, and $\mathrm{A}_{4}$ (peak, $P<0.005$; AUC, $P<0.001$ ) to ACTH tests were significantly higher in the patients with IHA than in the control subjects (Table 2). There was a significant correlation between the basal DHEAS levels, peak 11-S, and $\mathrm{AUC}_{11-\mathrm{S}}$ in response to $\mathrm{ACTH}$-stimulation test in patients with IHA $(P<0.005, r, 0.6)$ (Fig. 1).

None of the control subjects showed glucose intolerance after OGTT. Four (16.6\%) patients had glucose intolerance (one diabetes mellitus (DM); three IGT). Only the patient with DM had a BMI $>30 \mathrm{~kg} / \mathrm{m}^{2}$ 
Table 1 Clinical and basal hormonal characteristics of the patients with IHA and control subjects. Values are means \pm s.D. For each value, parenthesis shows median and the ranges respectively.

\begin{tabular}{|c|c|c|c|}
\hline & IHA group $(n=24)$ & Control group $(n=20)$ & $P$-value \\
\hline Age (years) & $27 \pm 6.1(26)(18-38)$ & $29.2 \pm 6.7(28.5)(19-40)$ & NS \\
\hline Body mass index (BMI) $\left(\mathrm{kg} / \mathrm{m}^{2}\right)$ & $26.2 \pm 7.2(24.3)(17.4-41.2)$ & $24.6 \pm 4.0(24.2)(17.8-31.1)$ & NS \\
\hline Ferriman-Gallwey score & $15.8 \pm 4.6(15)(9-27)$ & $2.2 \pm 1.1(2)(0-5)$ & $<0.001$ \\
\hline Cortisol ( $\mu \mathrm{g} / \mathrm{dl})$ & $14.6 \pm 5.2(12.7)(8.3-30.2)$ & $15.1 \pm 7.6(13.4)(7.9-29)$ & NS \\
\hline Follicle-stimulating hormone (FSH) (mIU/ml) & $5.6 \pm 2.0(5.4)(1.3-11.7)$ & $6.4 \pm 2.6(5.5)(1.4-12.6)$ & NS \\
\hline Luteinizing hormone (LH) $(\mathrm{mlU} / \mathrm{ml})$ & $4.9 \pm 2.0(4.8)(1.6-10.6)$ & $5.2 \pm 2.0(4.9)(2.7-9.4)$ & NS \\
\hline Estradiol (pg/ml) & $88.7 \pm 47.1(76)(30.6-203)$ & $81.3 \pm 36.4(80)(33.0-193)$ & NS \\
\hline $\mathrm{LH} / \mathrm{FSH}$ & $0.9 \pm 0.4(0.8)(0.2-2.2)$ & $0.9 \pm 0.4(0.8)(0.4-2.1)$ & NS \\
\hline 17-OHP (ng/ml) & $1.8 \pm 1.1(1.3)(0.6-5.1)$ & $1.5 \pm 0.9(1.1)(0.5-4.4)$ & NS \\
\hline $11-\mathrm{S}(\mathrm{ng} / \mathrm{ml})$ & $2.4 \pm 1.2(2.0)(0.8-6.4)$ & $1.8 \pm 0.8(1.7)(0.8-4)$ & NS \\
\hline $\begin{array}{l}\text { Serum sex hormone-binding globulin (SHBG) } \\
(\mathrm{nmol} / \mathrm{l})\end{array}$ & $40.3 \pm 37.8(32)(8.9-198)$ & $49.5 \pm 40.9(35)(12-170)$ & NS \\
\hline Androstenedione (ng/ml) & $3.1 \pm 1.6(2.8)(1.0-9.3)$ & $1.7 \pm 0.6(1.4)(0.6-2.8)$ & $<0.001$ \\
\hline Total testosterone (ng/dl) & $69.6 \pm 40.8(60)(13-192)$ & $37.9 \pm 21.8(35)(8-78)$ & $<0.005$ \\
\hline Free testosterone $(\mathrm{pg} / \mathrm{ml})$ & $3.0 \pm 1.9(2.4)(0.6-8.4)$ & $1.8 \pm 0.7(1.9)(0.8-3.2)$ & $<0.05$ \\
\hline DHEAS $(\mathrm{ng} / \mathrm{ml})$ & $4576 \pm 2116(4249)(747-9240)$ & $1925 \pm 1127(1740)(356-4990)$ & $<0.001$ \\
\hline
\end{tabular}

NS, not significant; 17-OHP, 17-hydroxyprogesterone; 11-S, 11-deoxycortisol, DHEAS, dehydroepiandrosterone sulfate.

and the subjects with glucose intolerance had a BMI $<30 \mathrm{~kg} / \mathrm{m}^{2}$. Fasting glucose, basal insulin, $\mathrm{AUC}_{\text {insulin }}$ levels, and HOMA scores were similar between patient and control groups, while the $\mathrm{AUC}_{\text {glucose }}$ value was higher in women with IHA $(P<0.01)$ (Table 3$)$. Analyses after omitting the patients with IGT showed that there was still a significant $(P<0.01)$ difference in terms of $\mathrm{AUC}_{\text {glucose }}$ value between the patients (13 656 2474 ) and controls (11 423 \pm 1810$)$.

Only one patient $(3.8 \%)$ was diagnosed as $21-\mathrm{OH}-\mathrm{de}-$ ficient NCAH after ACTH. The patients with 21-OH deficiency and DM were excluded from statistical analyses.

\section{Discussion}

Androgen excess is one of the most common endocrine disorders of reproductive-aged women and affects nearly $7 \%$ of the population $(1,3,11)$. Disorders that result in androgen excess include some specific ones such as androgen-secreting tumors and NCAH. On the other hand, a number of androgen excess disorders are diagnosed by exclusion such as PCOS. Although hirsutism is the most common feature of androgen excess, it is well known that not all patients with hirsutism have overt androgen excess as in idiopathic hirsutism $(12,13)$. We have previously shown that a number of patients with hirsutism had hyperandrogenemia with normal ovaries and regular cycles. These patients exhibited similar basal FSH, LH, testosterone, androstenedione, and DHEAS levels PCOS to subjects, however, they did not meet the diagnostic criteria for PCOS. We have named these patients as IHA (3). The underlying mechanism(s) in these patients is not clearly known. Therefore, we aimed to investigate their adrenal gland functions. It has been suggested that adrenal stimulation with ACTH has been the principal challenge test for estimating the relative activity of adrenocortical enzymes $(8,14)$. None of the patients, except one, met the criteria of NCAH (21-hydroxylase) in response to ACTH test. We have found that ACTH-stimulated androstenedione, 11-S, and DHEAS levels were

Table 2 ACTH-stimulated hormone levels and their AUC values in patients with IHA and in the control women. Values are means \pm s.D. For each value, parenthesis shows median and the ranges respectively.

\begin{tabular}{lccc}
\hline & IHA group $(n=24)$ & Control group $(n=20)$ & P-value \\
\hline Peak cortisol $(\mu \mathrm{gg} / \mathrm{dl})$ & $26.4 \pm 5.6(26.6)(16.2-35.0)$ & $25.8 \pm 5.3(25.4)(15.9-35.2)$ & $\mathrm{NS}$ \\
Peak 17-OHP $(\mathrm{ng} / \mathrm{ml})$ & $3.9 \pm 1.2(3.5)(1.6-6.4)$ & $3.9 \pm 1.6(3.7)(2.1-9.6)$ & $\mathrm{NS}$ \\
Peak 11-S $(\mathrm{ng} / \mathrm{ml})$ & $4.5 \pm 1.8(4.0)(1.4-9.1)$ & $3.6 \pm 1.0(3.3)(2.4-6.1)$ & $<0.05$ \\
Peak DHEAS $(\mathrm{ng} / \mathrm{ml})$ & $5657 \pm 2760(5677)(895-10621)$ & $3171 \pm 3269(2513)(816-15756)$ & $<.005$ \\
Peak $A_{4}(\mathrm{ng} / \mathrm{ml})$ & $3.8 \pm 1.3(3.3)(2.2-7.4)$ & $2.8 \pm 1.1(2.9)(1.4-6.9)$ & $\mathrm{NS}$ \\
AUC $_{\text {cortisol }}(\mu \mathrm{g} / \mathrm{dl} \times 60 \mathrm{~min})$ & $1320 \pm 295(1341)(866-1878)$ & $1212 \pm 312(1159)(776-1779)$ & $\mathrm{NS}$ \\
AUC $_{17-O H P}(\mathrm{ng} / \mathrm{ml} \times 60 \mathrm{~min})$ & $186 \pm 67(177)(70.6-353)$ & $177 \pm 78(161)(95-425)$ & $<0.05$ \\
AUC $_{11-S}(\mathrm{ng} / \mathrm{ml} \times 60 \mathrm{~min})$ & $211 \pm 85(194)(76.2-399)$ & $168 \pm 42(162)(102-261)$ & $<0.005$ \\
AUC $_{\text {DHEAS }}(\mathrm{ng} / \mathrm{ml} \times 60 \mathrm{~min})$ & $266490 \pm 124934(274800)$ & $(116872)(44460-708780)$ & $134 \pm 54(123)(63-306)$
\end{tabular}

NS, not significant; 17-OHP, 17-hydroxyprogesterone; 11-S, 11-deoxycortisol; DHEAS, dehydroepiandrosterone sulfate; AUC, area under the curve; $\mathrm{A}_{4}$, androstenedione. 

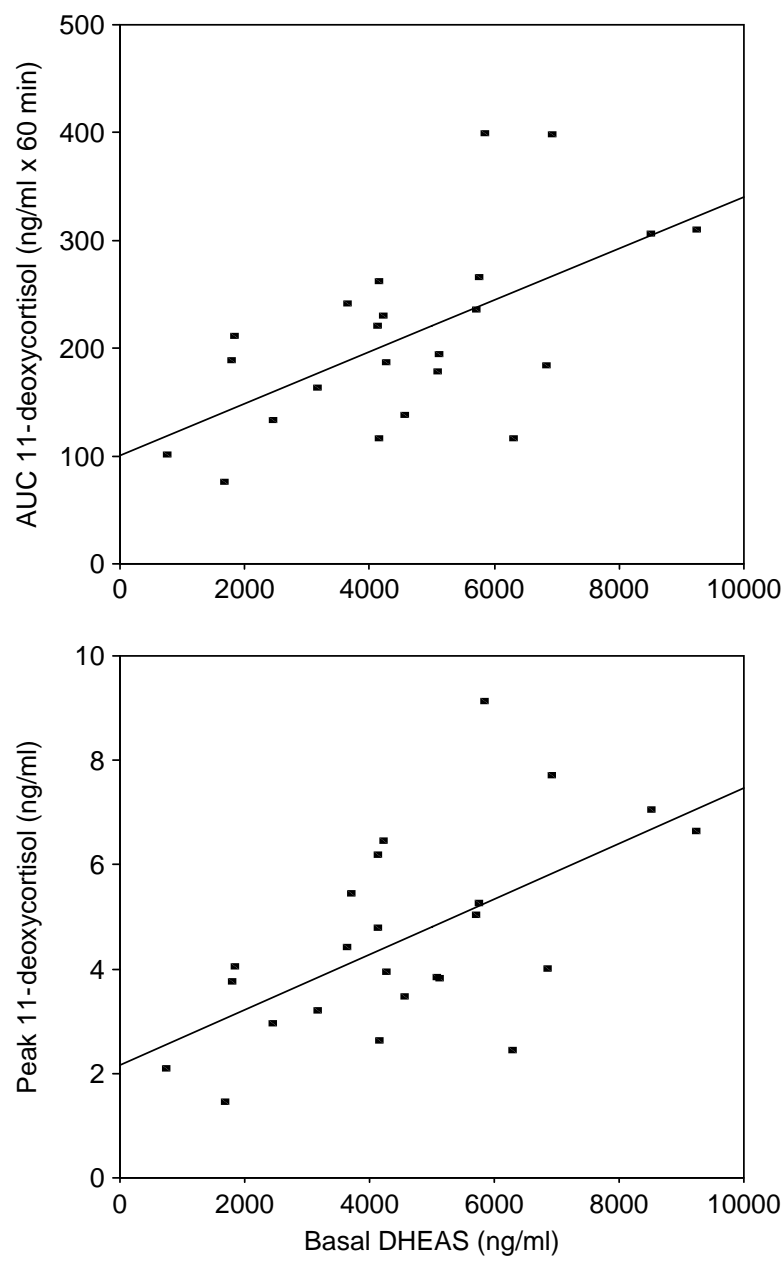

Figure 1 Correlation between basal DHEAS and AUC (upper panel) and peak (lower panel) levels of 11-deoxycortisol in patients with $\mathrm{IHA}$ in response to ACTH-stimulation test $(P<0.005, r=0.6)$.

significantly higher in the patients with IHA than in the control group. Our results also showed that DHEAS levels were correlated with the peak and AUC 11-S values. These data suggest that patients with IHA have an increased adrenal androgen secretion. This is in accordance with our previous report (15) demonstrating the presence of an alternate pathway in the adrenal gland in women with PCOS, resulting in an increase in adrenal androgens. Similar mechanism may operate in the patients with IHA.
A similar kind of patient has been evaluated recently by Azziz et al. (1). They suggested that 7\% of the patients had hyperandrogenemia but with normal ovulation and represent a diagnostic conundrum. The authors suggested that these women were less likely to be infertile, reflecting their normal ovulatory status and have higher DHEAS levels than PCOS patients. They think that these patients more closely resemble idiopathic hirsutism than PCOS patients and suggest that they represent predominantly adrenal androgen excess. Our data further support the suggestion of Azziz et al. in terms of adrenal hyperactivity in these patients. Recently, Carmina et al. (16) also used this new entity, IHA. They have re-evaluated the diagnosis of PCOS in 290 hyperandrogenic women and divided them into three groups, such as classic PCOS (patients with hyperandrogenism and chronic anovulation), ovulatory PCOS (patients with hyperandrogenism and polycystic ovaries but with normal ovulatory cycles), and patients with idiopathic hyperandrogenism (patients with hyperandrogenism but with ovulatory cycles and normal ovaries). They suggest that by using the new European Society for Human Reproduction and Embryology/American Society for Reproductive Medicine criteria (17) for the diagnosis of PCOS, $88 \%$ of the hyperandrogenic women of that study were considered to have PCOS and $12 \%$ had IHA (16). The authors suggest that three phenotypes of PCOS exist including IHA according to the ESHRE/ASRM criteria. In contrast, we think that IHA is not a subtype of PCOS, since patients with IHA do not fit the two out of three criteria (anovulation, hyperandrogenemia, or hyperandrogenism, polycystic ovarian changes) for the diagnosis of PCOS. Our data suggest that it is a kind of functional adrenal hyperactivity, for which the cause is unknown.

The association of carbohydrate metabolism abnormalities with androgen excess disorders, particularly PCOS, is a well-defined entity. We have also shown an increased prevalence of glucose intolerance in women with idiopathic hirsutism, who actually have normal serum androgen levels (12). From another point of view it has been shown that hyperinsulinemia during OGTT might activate the androgen pathway of the adrenal cortex (18). In the present study, we have also investigated the prevalence of glucose intolerance and

Table 3 The insulin resistance parameters of women with IHA and control subjects. Values are means \pm s.D. For each value, parenthesis shows median and the ranges respectively.

\begin{tabular}{lccc}
\hline & Patients with IHA $(n=24)$ & Control subjects $(n=20)$ & $\boldsymbol{P}$ \\
\hline FPG $(\mathrm{mg} / \mathrm{dl})$ & $75 \pm 8.0(75)(63-91)$ & $76 \pm 13(77.5)(62-108)$ & $\mathrm{NS}$ \\
Basal insulin $(\mathrm{mU} / \mathrm{l})$ & $9.0 \pm 4.9(9.1)(1.7-21)$ & $11 \pm 7(11.4)(0.8-28)$ & $\mathrm{NS}$ \\
AUC glucose $_{(\mathrm{mg} / \mathrm{dl} \times 120 \mathrm{~min})}$ & $13868 \pm 2472(14115)(9675-17820)$ & $11423 \pm 1810(10890)(8895-15390)$ & $<0.01$ \\
AUC ${ }_{\text {insulin }}(\mathrm{mU} / \mathrm{l} \times 120 \mathrm{~min})$ & $7071 \pm 5024(7104)(600-24519)$ & $5779 \pm 3302(4654)(861-14770)$ & $\mathrm{NS}$ \\
HOMA-IR & $1.7 \pm 0.9(1.5)(0.3-3.8)$ & $2.1 \pm 1.5(1.9)(0.1-5.9)$ & $\mathrm{NS}$
\end{tabular}

NS, not significant; FPG, fasting plasma glucose; AUC, area under the curve; HOMA-IR, HOMA of insulin resistance. 
the presence or absence of insulin resistance in patients with IHA. We found that $16.6 \%$ of the patients had glucose intolerance and a higher $\mathrm{AUC}_{\text {glucose }}$ value. Analyses after omitting the patients with IGT showed that there was still a significant difference in terms of $\mathrm{AUC}_{\text {glucose }}$ value between the patients and controls. Although, $\mathrm{AUC}_{\text {insulin }}$ value, fasting glucose, basal insulin, and HOMA scores were similar between patients and control groups, increased glucose response to OGTT suggest that there is a tendency towards insulin resistance in patients with IHA. Hyperandrogenemia per se may have a role in the higher prevalence of glucose intolerance in these patients. Similarly, Carmina et al. (16) reported that 26\% of the patients with IHA had insulin resistance, thereby suggesting the existence of mild metabolic and hormonal abnormalities.

In conclusion, our data suggest that adrenal androgen excess may play a role in patients with IHA. Although we did not investigate the ovarian functions in detail, the adrenal glands may be the major sources of androgens in patients with IHA. These patients exhibit increased prevalence of glucose intolerance. Further studies including the assessment of ovarian function are needed to clarify the pathophysiology of IHA.

\section{References}

1 Azziz R, Sanchez LA, Knochenhauer ES, Moran C, Lazenby J, Stephens KC, Taylor K \& Boots LR. Androgen excess in women: experience with over 1000 consecutive patients. Journal of Clinical Endocrinology and Metabolism 200489 453-462.

2 Unluhizarci K, Sahin Y \& Kelestimur F. The evaluation and treatment of hirsute women. Women's Health 20051 429-435.

3 Unluhizarci K, Gokce C, Atmaca H, Bayram F \& Keleştimur F. A detailed investigation of hirsutism in a Turkish population: idiopathic hyperandrogenemia as a perplexing issue. Experimental and Clinical Endocrinology Diabetes 2004112 1-6.

4 Hatch R, Rosenfield RL, Kim MH \& Tredway D. Hirsutism: implications, etiology and management. American Journal of Obstetrics and Gynecology 1981140 815-830.

5 Romaquera J, Moran C, Diaz-Montes TP, Hines GA, Cruz RI \& Azziz R. Prevalence of 21-hydroxylase deficient nonclassic adrenal hyperplasia and insulin resistance among hirsute women from Puerto Rico. Fertility and Sterility 200074 59-62.
6 Unluhizarci K, Kelestimur F, Guven M, Bayram F \& Colak R. The value of low dose $(1 \mu \mathrm{g})$ ACTH stimulation test in the investigation of non-classic adrenal hyperplasia due to $11 \beta$ hydroxylase deficiency. Experimental and Clinical Endocrinology Diabetes 2002 $110381-385$

7 Kelestimur F, Sahin Y, Ayata D \& Tutus A. The prevalence of nonclassic adrenal hyperplasia due to $11 \beta$ hydroxylase deficiency among hirsute women in a Turkish population. Clinical Endocrinology $1996 \mathbf{4 5} 381-384$.

8 Azziz D, Dewailly D \& Owerbach D. Nonclassic adrenal hyperplasia: current concepts. Journal of Clinical Endocrinology and Metabolism 199478 810-815.

9 Bonora E, Targher G, Alberiche M, Bonadonna RC, Saggiani F, Zenere MB, Monauni $\mathrm{T}$ \& Muggeo M. Homeostasis model assessment closely mirrors the glucose clamp technique in the assessment of insulin sensitivity. Diabetes Care 200023 57-63.

10 The expert committee on the diagnosis and classification of diabetes mellitus. Report of the expert committee on the diagnosis and classification of diabetes mellitus. Diabetes Care 199821 S5-S19.

11 Keleştimur F. Hirsutism of adrenal origin in adolescents: consequences in adults. Journal of Pediatrical Endocrinology and Metabolism 200114 1309-1315.

12 Unlühizarci K, Karababa Y, Bayram F \& Kelestimur F. The investigation of insulin resistance in patient with idiopathic hirsutism. Journal of Clinical Endocrinology and Metabolism 2004 89 2741-2744.

13 Azziz R, Carmina E \& Sawaya ME. Idiopathic hirsutism. Endocrine Reviews 200021 347-362.

14 Azziz R, Hincapie LA, Knochenhauer ES, Dewailly D, Fox L \& Boots LR. Screening for 21-hydroxylase-deficient nonclassic adrenal hyperplasia among hyperandrogenic women: a prospective study. Fertility and Sterility 199972 915-925.

15 Kelestimur F \& Sahin Y. Alternate pathway 17,20-lyase enzyme activity in the adrenals is enhanced in patients with polycystic ovary syndrome. Fertility and Sterility 199971 1075-1078.

16 Carmina E, Chu MC, Longo RA, Rini GB \& Lobo RA. Phenotypic variation in hyperandrogenic women influences the findings of abnormal metabolic and cardiovascular risk parameters. Journal of Clinical Endocrinology and Metabolism $2005902545-2549$.

17 The Rotterdam ESHRE/ASRM sponsored PCOS Consensus Workshop Group. Revised 2003 consensus on diagnostic criteria and long term health risks related to polycystic ovary syndrome. Fertility and Sterility 200481 19-25.

18 Vasarhelyi B, Bencsik P, Tereszl A, Bardoczy Z, Tulassay T \& Szathmari M. The effect of physiologic hyperinsulinemia during an oral glucose tolerance test on the levels of dehydroepiandrosterone (DHEA) and its sulfate (DHEAS) in healthy young adults born with low and with normal birth weight. Endocrine Journal 20036 689-695.

Received 14 February 2006

Accepted 18 May 2006 